\title{
Hemodynamics in the retinal vasculature during the progression of diabetic retinopathy
}

Francesco Calivá ${ }^{1 *}$, Georgios Leontidis ${ }^{1 *}$, Piotr Chudzik $^{1}$, Andrew Hunter ${ }^{1}$, Luca Antiga $^{2}$, Bashir Al-Diri ${ }^{1}$

${ }^{1}$ School of Computer Science, University of Lincoln, Lincoln, United Kingdom, ${ }^{2} O R O B I X$ srl, Bergamo, Italy

\section{Abstract}

Purpose: Several studies have established, using various measurement modalities, that progression from diabetes to diabetic retinopathy (DR) is associated with changes in hemodynamics or measurable vascular geometry. In this study, we take vessel measurements from standard fundus images and estimate hemodynamic parameters (which are not directly observable) using a simple hemodynamic model. We show that there are statistically significant changes in some estimated hemodynamic parameters associated with the development of DR.

Methods: A longitudinal study of 24 subjects was conducted. For each subject, four fundus images were used, taken annually during the three years before the appearance of DR and in the first year of DR. A venous and arterial vascular bifurcation, consisting each of a parent vessel and two child branches, was extracted and at the branching nodes a zero dimensional model estimated the fluid dynamic conditions in terms of volumetric blood flow, blood flow velocity, nodal pressure, wall shear stress, and Reynolds number. These features were statistically analyzed using linear mixed models.

Results: A number of parameters, primarily venous, showed significant change with the development of DR, including early change two years before the onset of DR. A large proportion of overall variance is accounted for by individual patient differences, making progressive study essential.

Conclusion: This is the first paper to demonstrate that hemodynamic feature estimates extracted from standard fundus images are sensitive to progression from

Correspondence: Georgios Leontidis, School of Computer Science, University of Lincoln, Brayford Pool Campus, LN67TS, UK.

E-mail: gleontidis@lincoln.ac.uk 
diabetes to DR. In our future work, we aim to test whether the variations in hemodynamic conditions are predictive of progression prior to the appearance of retinal lesions.

Keywords: diabetic retinopathy (DR), retinal biomarkers, retinal microcirculation, retinal trees, retinal vascular geometry

\section{Introduction}

Diabetic retinopathy (DR) is characterized by lesions and vascular abnormalities, which include but are not limited to microaneurysms, hemorrhages, cotton wool spots, exudates (bright spots), venous beading, intraretinal microvascular abnormalities, neovascularization, and loop and fibrous proliferation. ${ }^{1,2}$ The World Health Organization (WHO) suggests that between 1980 and 2014, the prevalence of diabetes increased from $4.7 \%$ to $8.5 \%$. In 2012, 1.5 million deaths were directly associated with diabetes and another 2.2 million deaths were linked to high blood glucose levels. ${ }^{3}$ Early diagnosis of DR would allow clinicians to suggest patient-specific treatment plans for patients affected by diabetes and ensure efficient monitoring of the disease.

\subsection{Retinal vascular geometry and hemodynamics}

Hemodynamic factors such as perfusion pressure, vascular resistance, and blood viscosity together with the vascular geometry determine the distribution of blood flow to the retina, ${ }^{4}$ although the mechanisms linking hemodynamic alterations to systemic or ocular diseases are not completely understood yet. ${ }^{5,6}$ Technologies such as the Retinal Vessel Analyzer, ${ }^{7}$ Doppler-based techniques, ${ }^{8,9}$ and Retinal Function Imager ${ }^{10}$ allow non-invasive study of retinal haemodynamics.

Several studies have (separately) investigated the effects of diabetes on retinal vascular geometry and hemodynamics. Burgansky et al. studied the effect of DR on the arterial blood flow velocity, which was found to be slower in patients with DR. ${ }^{11}$ Grunwald et al. recruited a group of patients which had been affected by diabetes for less than four years, and age-matched them with normal subjects. The total measured blood flow rate in the diabetic patients was significantly higher compared to the normal subjects. ${ }^{12}$ Leontidis et al. observed significant changes in fractal dimension and arterial and venular widths. ${ }^{13-15}$ Kifley et al. observed a relationship between the increase of DR severity and the widening of retinal venular width. ${ }^{16}$ The Wisconsin epidemiological study showed that a correlation between the widening of retinal venulae and the progression of retinopathy exists. ${ }^{17}$ Yang et al. observed only wider venular caliber in the presence of DR. ${ }^{18}$ 


\subsection{Novelty of the present study}

In this paper, we introduce a 0-D model for the estimation of hemodynamic parameters from standard fundus images, and evaluate whether alteration of these estimated parameters is associated with the progression of diabetes to DR. This is potentially a more informative route to the detection of vascular disease than direct correlation from geometry measurements to disease, since the hypothesized cause of changes in vessel geometry is reaction to haemodynamic factors. Taking into account that vascular geometry may be influenced by multiple factors including age, duration of diabetes, lifestyle, gender, and phenotypic variation, the progression of the disease was investigated over a four-year period, culminating in the first diagnosis of DR by analyzing fundus images from a cohort of 24 patients. The main contribution of this study is to establish that there are significant associations between estimated hemodynamic features and disease progression; to the best of the authors' knowledge, this has never been attempted before.

\section{Methods and results}

A longitudinal study of 24 patients was conducted. Ninety-six fundus photographs (FP) $(1700 \times 1700$ pixels) were selected from the diabetic screening service of the Pilgrim Hospital in Boston, UK. For each patient, four photographs were captured annually during the three years before DR and in the first year of DR.

\subsection{Retinal blood flow mechanics}

Blood vessels were segmented out using the Ribbon of Twins (RoT) method. ${ }^{19}$ The RoT technique is an active contour method that uses morphological filters to identify the centerline of a vessel, and exhibits excellent performance in extracting edges. In each selected fundus image, one arterial and one venous bifurcation (Fig. 1a) were reconstructed using a semi-supervised tool; ${ }^{20}$ a bifurcation consists of three vessel segments, the parent and its two children. For each patient, the same venous and arterial bifurcations were selected throughout the four-year period, allowing a meaningful analysis of the changes.

\subsection{O-D vascular model}

A zero-dimensional model was designed to simulate the fluid dynamics within the network. As the diameter of the vessels was below $200 \mu \mathrm{m}$, the hemodynamics would be in the microcirculation regime, ${ }^{21,22}$ where Fahraeus and Fahraeus-Lindqvist effects as well as plasma skimming are crucial in determining the distribution of the hematocrit and blood flow velocity profile. ${ }^{23,24}$ However, for simplicity our model treats blood as a Newtonian fluid. ${ }^{25}$ Blood flow was modelled as $0-D$, and thus the mean blood flow velocity was used. Blood flow was assumed to follow Hagen-Poiseuille's law $(\mathrm{H}-\mathrm{P})$. H-P flow describes the relationship between the pressure drop $(\Delta P)$ and 

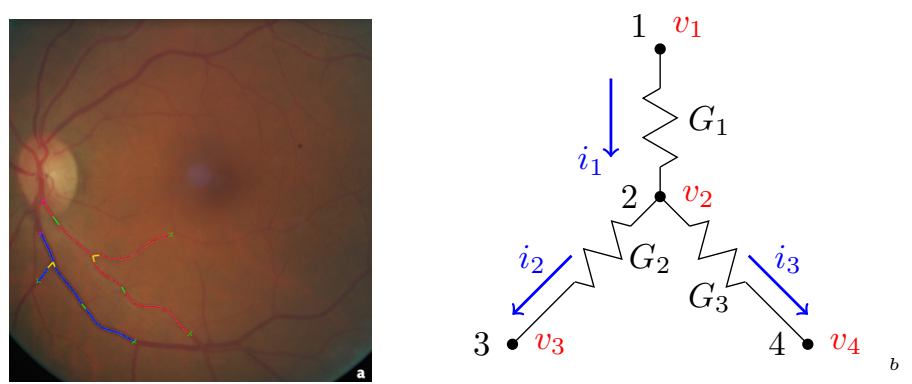

Fig. 1. (a) An arterial (red) and venous (blue) tree. Bifurcations are colored yellow. The green dashes represent the connection between the segments in which a blood vessel is split by the segmentation algorithm. (b) A lumped electric circuit. Nodes are denoted by the numbers 1, 2, 3 and 4 ; nodal voltages by $v_{i}$, with $i=1, \ldots, 4$; currents by $i_{k}$, with $k=1, \ldots, 3$; conductances by $G_{k}$, with $k=1, \ldots, 3$. The blue arrows represent the current flow direction.

the blood flow through a tube $\left(\Delta P=R_{i} \cdot Q_{i}\right)$, under the assumptions of a stiff, straight, and uniform tube; a Newtonian fluid; a circular cross-section; a laminar, steady (i.e., non pulsatile) flow with null velocity at the wall (no-slip condition). $R_{i}=$ $\frac{8 \mu L_{i}}{\pi r_{i}^{4}}$ is the resistance that the blood encounters when flowing in the $i^{-t h}$ tube of radius $r_{i}$ and length $L_{i}$, with blood viscosity of $\mu=0.04 P ; G_{i}=\frac{\pi r_{i}^{4}}{8 \mu L_{i}}$ is the conductance of the vessel. The retinal network was studied with an electrical lumped elements model. The conservation of mass was assumed at the branching nodes. Hence, the inflow of blood at the inlet matched the outflow from the bifurcation. Similar to Kirchhoff's conservation law of electric current at each node, for the $j^{-t h}$ branching point, the relationship $\sum_{k=p, d_{1}, d_{2}} Q_{j, k}=0$ holds, where $p, d_{1}, d_{2}$ refer to the parent branch and the two child branches of the bifurcation, respectively. ${ }^{26}$ By combining $\mathrm{H}$ P's law and the segment's conductance term, an explicit equation for $Q$ was obtained $\left(\Delta Q_{j k}=G_{j k} \cdot \Delta P_{j k}\right)$ in which the subscripts $j$ and $k$ refer to the node of the circuit (e.g., Fig. 1b). For the circuit in Figure 1b, the pressure value at each node was computed by applying the conservation law, and solving the system of linear equations $Q_{j k}=\Delta P_{j k} G_{j, k}$. Figure 1b shows an example of a circuit, which represents a tree displayed in Figure 1a. By applying Kirchhoff's law at each node of the system in this circuit, Equation (1) was obtained; its solution provided the pressure values at each node of the circuit.

$$
\left[\begin{array}{llll}
1 & 0 & 0 & 0 \\
G_{1} & -\left(G_{1}+G_{2}+G_{3}\right) & G_{3} & 0 \\
0 & 0 & 1 & 0 \\
0 & 0 & 0 & 1
\end{array}\right] \cdot\left[\begin{array}{l}
P_{1} \\
P_{2} \\
P_{3} \\
P_{4}
\end{array}\right]=\left[\begin{array}{l}
P_{\text {in }} \\
0 \\
P_{\text {out } 3} \\
P_{\text {out } 4}
\end{array}\right]
$$




\subsubsection{Boundary conditions (BCs)}

The fluid dynamic conditions in the arterial and venular trees, which included one bifurcation, were computed. Two experiments were carried out. In the first, BCs were set up in terms of pressure; in the second, in terms of blood flow.

1.Pressure boundary conditions: At the inlet (parent of the bifurcation), a pressure value of $40 \mathrm{mmHg}$ was specified. This choice reflects the hydrostatic and frictional pressure losses from the aorta to the central retinal artery (CRA). At the outlet (children of the bifurcation), a pressure slightly higher than $15 \mathrm{mmHg}$, equivalent to the intraocular pressure (IOP) in normal conditions, was enforced to prevent vessel collapse.

2.Flow boundary conditions: At the inlet, the flow rate $Q$ was computed as $Q_{i}\left(r_{i}\right)=$ $\pi r_{i}^{2} \overline{v_{i}}$. For the arterial trees, the enforced $Q$ was computed based on a mean velocity blood flow $(\bar{v}=6.4 \mathrm{~cm} / \mathrm{s})$, measured in the CRA by Fuchs et al. using color Doppler imaging, ${ }^{27}$ and a CRA diameter of $166 \mu \mathrm{m}$ suggested by Pemp et al. ${ }^{28}$ In the venous trees, the literature reports varying values for central retinal vein (CRV) blood flow velocity and diameter. ${ }^{28,29}$ The mean velocity blood flow used in this work was $\bar{v}=$ $3.6 \mathrm{~cm} / \mathrm{s}$, and CRV diameter $D=210 \mu \mathrm{m}$. At the outlet, the blood flow was enforced by assuming that blood flow velocity is constant at the bifurcation point. Therefore, with regard to the equation which expresses the relationship between vessel caliber, blood flow rate, and blood flow velocity, in each of the branches (i.e., parent and children), $Q$ depends only on the vessel caliber squared and was computed as $Q_{j}=Q_{i n} \cdot \frac{r_{j}^{2}}{\sum_{k \in \text { Outlet }} r_{k}^{2}}$.

\subsection{Hemodynamic parameter estimates}

The nodal pressure $(P)$ was estimated by solving the system in Equation (1). In each vessel, four parameters were estimated. The flow rate $(Q)$ was computed by solving Poiseuille's law equation. The wall shear stress (WSS) was computed in each $i^{-t h}$ vessel as $W S S_{i}=\frac{32 \mu Q_{i}}{\pi D_{i}^{3}}$. The blood flow velocity $(v)$ was computed in each vessel by rearranging and solving the equation which expressed the relationship between $\overline{v_{i}}$, $Q$ and $r$. The Reynolds number $(R e)$ was computed as $R e=\frac{\bar{v} D \rho}{\mu}$, where $\rho=1.060$ $\mathrm{g} / \mathrm{mL}$ was the dynamic viscosity of the blood.

\subsection{Statistical analysis}

The statistical analysis relates the five estimated hemodynamic features, both venous and arterial, to the year of measurement (i.e., progression towards disease), while accounting for individual differences between patients. The analysis was performed using linear mixed effects models (LMM), ${ }^{30}$ in which fixed and random effects combine linearly with an error term to determine a response variable. LMMs are similar to the Analysis of Variance (ANOVA) test, but they are considerably more generalized, supporting post-hoc analysis and utilizing additional evaluation metrics, as can be seen 
in the model comparison part that follows. ${ }^{31}$ The LMM captures individual differences by estimating a different random intercept for each subject.

A "full" LMM was constructed for each feature, with the feature as the response variable, the year of measurement as the (independent) variable of fixed effects, and the subject identity as the (independent) variable of random effects. A second, "restricted" model was also constructed for each feature, with the subject identity as the sole independent (random effects) variable.

The models were used to investigate the relationship of the year of measurement to the features as follows. First, the full models were fitted using restricted maximum likelihood (REML) and the Welch-Satterthwaite estimation of $p$-values based on degrees of freedom ${ }^{32}$ ) calculated. Second, both the full and restricted models were fitted using Maximum Likelihood (ML), p-values calculated from the likelihood ratio between the predictions of the two models, and the Akaike information criteria (AICs) calculated for both models. The restricted models were used to calculate the intra-class correlation (ICC), which describes the proportion of the total variance that can be explained by the individual patient identity. Post-hoc comparisons were made to investigate the relationship between the year of measurement and the statistically significant features; a Tukey-test was used to identify which year group means differed. ${ }^{33}$ The analyses were carried out in a balanced design. Table 1 lists the results for all the estimated parameters with significant results $(p<0.05)$. The lower AIC values for the "full" models indicate superior performance to the "restricted" models, showing that the year of measurement is related to the reported features; the two p-values are alternative methods that indicate the statistical significance of this relationship. All five venous parameters show significant results, with stronger effects in the child-vessels, and wall shear stress and velocity particularly affected. In the arteries, effects are seen only in the volumetric blood flow and Reynold's number parameters in the child-vessels. The ICC values show that, particularly in the venous analysis, there is a high level of individual patient variation, so that the strategy of including the patient identity as a random effects factor in the context of a longitudinal study was crucial to the success of the model.

Table 2 presents the post-hoc analysis of the features, listing the year to year comparisons where a significant difference was detected, with associated p-values. For the venous features with the strongest effects (e.g., WSS, $v$, and Re in the first childvessel) a consistent pattern emerged indicating that the most significant effects occur between Year 3 and the other years: Year 2, Year 0 (DR onset) and Year 1, in that order. The dip in significance in Year 1 suggests there may be a complex development of the hemodynamics features. Given the difference in methods and modalities, a direct comparison could not be made with other studies, although we note that elsewhere (e.g., Bursell et al., Grunwald et al., and Feke et al.), ${ }^{34-36}$ contradictory findings on blood flow rates in normal and diabetic patients have been reported. The significant difference in Year 3 to Year 2 is particularly interesting, as it is suggestive of the potential for early detection of DR onset. In the arterial network, the smaller number of significant features are most consistently affected between Year 3 and Year 1. 
Table 1. The hemodynamic features showing statistically significant relationship to year of measurement. Lower AIC values for "full" models indicate that the measurement year is significant; the two alternative $p$-value estimates confirm the significance of this difference. The ICC illustrates the proportion of variance accounted for by other differences between individual patients.

\begin{tabular}{|l|l|l|l|l|}
\hline Features & AIC $^{a}$ & p-(s) & p-(LR) & ICC $^{c}$ \\
\hline Veins_WSS_Parent & $550.7 / 554.3$ & 0.02 & 0.02 & 0.942 \\
\hline Veins_WSS_Child1 & $472.2 / 484.8$ & $<0.000$ & $<0.000$ & 0.917 \\
\hline _WSS_Child2 & $490.7 / 501.2$ & 0.001 & $<0.000$ & 0.901 \\
\hline Veins_Q_Child1 & $201.4 / 204.1$ & 0.05 & 0.034 & 0.955 \\
\hline Veins_Q_Child2 & $201.4 / 204$ & 0.04 & 0.033 & 0.915 \\
\hline Veins_v_Parent & $335.5 / 339.2$ & 0.024 & 0.02 & 0.833 \\
\hline Veins_v_Child1 & $231.9 / 247.2$ & $<0.000$ & $<0.000$ & 0.896 \\
\hline Veins_v_Child2 & $247.5 / 256.1$ & 0.003 & 0.002 & 0.915 \\
\hline Veins_Re_Parent & $693.4 / 696.8$ & 0.027 & 0.024 & 0.922 \\
\hline Veins_Re_Child1 & $593.9 / 610.4$ & $<0.000$ & $<0.000$ & 0.892 \\
\hline Veins_Re_Child2 & $608.3 / 611$ & 0.04 & 0.034 & 0.944 \\
\hline Veins_Pressure & $444.3 / 448.2$ & 0.02 & 0.017 & 0.833 \\
\hline Arteries_Q_Child1 & $106.2 / 111.5$ & 0.012 & 0.01 & 0.398 \\
\hline Arteries_Q_Child2 & $105.1 / 110.4$ & 0.011 & 0.009 & 0.399 \\
\hline Arteries_Re_Child2 & $800.1 / 804.5$ & 0.017 & 0.015 & 0.622 \\
\hline
\end{tabular}

${ }^{a}$ Full/restricted. ${ }^{b}$ P-value (Satterthwaite approximation). ${ }^{c}$ P-value (likelihood ratio).

\section{Conclusions and future perspectives}

In this paper, a number of retinal hemodynamic parameters (nodal pressure, volumetric blood flow, wall shear stress, blood flow velocity, and Reynolds number) were estimated using a 0-D model on measurements extracted from selected vessel bifurcations on fundus images. The estimated parameters were analyzed over a period of three years of progression from diabetes to DR within a group of 24 subjects. The results indicate that there are significant alterations to hemodynamic parameter estimates associated with detectable changes to vessel geometry, particularly in the venular network. These are particularly pronounced between the earliest year studied (three years before DR onset) and subsequent years.

In future work, we will utilize a larger data set to verify these findings, include a control sample to allow a full retrospective study, further investigate the evolution of hemodynamic features during disease progression, and extend the analysis to full vascular trees that include multiple bifurcations. We will also validate the 0 -D model predictions on functional images that include direct measurement of hemodynamic 
Table 2. Post-hoc analysis of the haemodynamic features, showing significant year to year differences. Differences from Year 3 to other years predominate.

\begin{tabular}{|l|l|l|}
\hline Features & Significant Hypotheses & P-values \\
\hline Veins_WSS_Parent & Year3-Year2 & 0.015 \\
\hline Veins_WSS_Child1 & Year3-Year2 & 0.002 \\
& Year3-Year1 & 0.019 \\
& Year3-DR & $<0.000$ \\
\hline Veins_WSS_Child2 & Year3-Year2 & $<0.000$ \\
& Year3-Year1 & 0.009 \\
& Year3-DR & 0.003 \\
\hline Veins_Q_Child1 & Year3-Year2 & 0.049 \\
& Year2-DR & 0.048 \\
\hline Veins_Q_Child2 & Year3-Year2 & 0.049 \\
& Year3-DR & 0.049 \\
\hline Veins_v_Parent & Year3-Year2 & 0.015 \\
\hline Veins_v_Child1 & Year3-Year2 & 0.001 \\
& Year3-Year1 & 0.008 \\
& Year3-DR & 0.001 \\
\hline Veins_v_Child2 & Year3-Year2 & 0.02 \\
& Year3-Year1 & 0.005 \\
& Year3-DR & 0.002 \\
\hline Veins_Re_Parent & Year3-Year2 & 0.018 \\
\hline Veins_Re_Child1 & Year3-Year2 & $<0.000$ \\
& Year3-Year1 & 0.009 \\
& Year3-DR & $<0.000$ \\
\hline Veins_Re_Child2 & Year3-DR & 0.017 \\
\hline Veins_Pressure & Year2-Year1 & 0.013 \\
& Year3-DR & 0.024 \\
\hline Arteries_Q_Child1 & Year3-Year1 & 0.006 \\
\hline Arteries_Q_Child2 & Year3-Year1 & 0.006 \\
\hline Arteries_Re_Child2 & Year3-Year2 & 0.035 \\
& Year3-Year1 & 0.024 \\
\hline & & \\
& & \\
& & \\
& &
\end{tabular}

features in addition to the standard fundus appearance. Finally, we will develop diagnostic models to predict DR onset from estimated hemodynamic features.

\section{Acknowledgements}

This research was made possible by a Marie Curie grant from the European Commission in the framework of the REVAMMAD ITN (Initial Training Research network), Project number 316990. The authors would also like to thank Mr P. Hopkins, United Lincolnshire Hospitals NHS Trust, Pilgrim Hospital, Diabetic Eye Screening Pro- 
gramme, Boston, United Kingdom, for letting us extract the fundus images.

\section{References}

1. Donnelly R, Horton E. Vascular complications of diabetes: current issues in pathogenesis and treatment. John Wiley \& Sons, 2008;

2. Leontidis G, Al-Diri B, Hunter A. Diabetic retinopathy: current and future methods for early screening from a retinal hemodynamic and geometric approach. Expert Review of Ophthalmology, 2014;9(5): 431-442.

3. WHO. Global report on diabetes. 2016;

4. Harris A. Atlas of ocular blood flow: vascular anatomy, pathophysiology, and metabolism. ButterworthHeinemann Medical, 2003;

5. Caprioli J, Coleman AL. Blood pressure, perfusion pressure, and glaucoma. American journal of ophthalmology, 2010;145(5): 704-712.

6. Weinreb RN, Harris A. Ocular blood flow in glaucoma. 2009,6.

7. Vilser W, Nagel E, Lanzl I. Retinal vessel analysis-new possibilities. Biomedizinische Technik/Biomedical Engineering, 2002;47(s1b): 682-685.

8. Nicolela MT, Hnik P, Drance SM. Scanning laser Doppler flowmeter study of retinal and optic disk blood flow in glaucomatous patients. American journal of ophthalmology, 1996;122(6): 775-783.

9. Wang Y, Lu A, Gil-Flamer J, Tan O, Izatt JA, Huang D. Measurement of total blood flow in the normal human retina using Doppler Fourier-domain optical coherence tomography. British Journal of Ophthalmology, 2009;93(5): 634-637.

10. Izhaky D, Nelson DA, Burgansky-Eliash Z, Grinvald A. Functional imaging using the retinal function imager: direct imaging of blood velocity, achieving fluorescein angiography-like images without any contrast agent, qualitative oximetry, and functional metabolic signals. Japanese journal of ophthalmology, 2009;53(4): 345-351.

11. Burgansky-Eliash Z, Nelson DA, Orly P, Lowenstein A, Grinvald A, Barak A. Reduced retinal blood flow velocity in diabetic retinopathy. Retina, 2010;30(5): 765-773.

12. Grunwald JE, Maguire AM, Dupont J. Retinal hemodynamics in retinitis pigmentosa. American journal of ophthalmology, 1996;122(4): 502-508.

13. Leontidis G, Al-Diri B, Wigdahl J, Hunter A. Evaluation of geometric features as biomarkers of diabetic retinopathy for characterizing the retinal vascular changes during the progression of diabetes. 2015 37th Annual International Conference of the IEEE Engineering in Medicine and Biology Society (EMBC). IEEE. 2015; 5255-5259.

14. Leontidis G, Al-Diri B, Hunter A. Summarising the retinal vascular calibres in healthy, diabetic and diabetic retinopathy eyes. Computers in biology and medicine, 2016;72, 65-74.

15. Leontidis $\mathrm{G}$. Early screening and diagnosis of diabetic retinopathy. PhD thesis. University of Lincoln, UK, 2016;

16. Kifley A, Wang JJ, Cugati S, Wong TY, Mitchell P. Retinal vascular caliber, diabetes, and retinopathy. American journal of ophthalmology, 2007;143(6): 1024-1026.

17. Klein R, Klein B, Moss SE, Davis MD, DeMets DL. The Wisconsin Epidemiologic Study of Diabetic Retinopathy: III. Prevalence and risk of diabetic retinopathy when age at diagnosis is 30 or more years. Archives of ophthalmology, 1984;102(4): 527-532.

18. Yang X, Deng Y, Gu H, Ren X, Lim A, Snellingen T, et al. Relationship of retinal vascular calibre and diabetic retinopathy in Chinese patients with type 2 diabetes mellitus: the Desheng Diabetic Eye Study. British Journal of Ophthalmology, 2016;

19. Al-Diri B, Hunter A, Steel D. An active contour model for segmenting and measuring retinal vessels. IEEE Transactions on Medical imaging, 2009;28(9): 1488-1497. 
20. Calivá F, Aletti M, Al-Diri B, Hunter A. A new tool to connect blood vessels in fundus retinal images. 37th Annual International Conference of the IEEE Engineering in Medicine and Biology Society (EMBC), 2015; 4343-4346.

21. Wong TY, Klein R, Klein BE, Meuer SM, Hubbard LD. Retinal vessel diameters and their associations with age and blood pressure. Investigative ophthalmology and visual science, 2003;44(11): 46444650.

22. Pournaras CJ, Rungger-Brändle E, Riva CE, Hardarson SH, Stefansson E. Regulation of retinal blood flow in health and disease. Progress in retinal and eye research, 2008;27(3): 284-330.

23. Pries AR, Neuhaus D, Gaehtgens P. Blood viscosity in tube flow: dependence on diameter and hematocrit. American Journal of Physiology-Heart and Circulatory Physiology, 1992;263(6): H1770-H1778.

24. Pries AR, Ley K, Claassen M, Gaehtgens P. Red cell distribution at microvascular bifurcations. Microvascular research, 1989;38(1): 81-101.

25. Aletti M, Gerbeau JF, Lombardi D. Modeling autoregulation in three-dimensional simulations of retinal hemodynamics. Journal for Modeling in Ophthalmology, 2015;1.

26. Causin P, Guidoboni G, Malgaroli F, Sacco R, Harris A. Blood flow mechanics and oxygen transport and delivery in the retinal microcirculation: multiscale mathematical modeling and numerical simulation. Biomechanics and modeling in mechanobiology, 2016;15(3): 525-542.

27. Fuchsjäger-Mayrl G, Polak K, Luksch A, Polska E, Dorner GT, Rainer G, et al. Retinal blood flow and systemic blood pressure in healthy young subjects. Graefe's archive for clinical and experimental ophthalmology, 2001;239(9): 673-677.

28. Pemp B, Cherecheanu AP, Garhofer G, Schmetterer L. Calculation of central retinal artery diameters from non-invasive ocular haemodynamic measurements in type 1 diabetes patients. Acta ophthalmologica, 2015;91(5): e348-e352.

29. Kaiser HJ, Schoetzau A, Flammer J. Blood flow velocity in the extraocular vessels in chronic smokers. British journal of ophthalmology, 1997;81(2): 133-135.

30. Bates D. Computational methods for mixed models. LME4: Mixed-Effects Modeling with R, 2014; 99118.

31. Krueger C, Tian L. A comparison of the general linear mixed model and repeated measures ANOVA using a dataset with multiple missing data points. Biological research for nursing, 2004;6(2): 151-157.

32. Satterthwaite FE. An approximate distribution of estimates of variance components. Biometrics bulletin, 1946;2(6): 110-114.

33. Tukey JW. Comparing individual means in the analysis of variance. Biometrics, 1949; 99-114.

34. Bursell SE, Clermont AC, Kinsley BT, Simonson DC, Aiello LM, Wolpert HA. Retinal blood flow changes in patients with insulin-dependent diabetes mellitus and no diabetic retinopathy. Investigative ophthalmology \& visual science, 1996;37(5): 886-897.

35. Grunwald JE, DuPont J, Riva CE. Retinal haemodynamics in patients with early diabetes mellitus. British journal of ophthalmology, 1996;80(4): 327-331.

36. Feke GT, Buzney SM, Ogasawara H, Fujio N, Goger DG, Spack NP, et al. Retinal circulatory abnormalities in type 1 diabetes. Investigative ophthalmology \& visual science, 1994;35(7): 2968-2975. 